\title{
A importância dos óleos essenciais na síntese verde de nanopartículas metálicas
}

\author{
The importance of essential oils in the \\ green synthesis of metallic nanoparticles
}

\author{
Milena Lima Guimarães ${ }^{1}$, Jarbas Freitas Amarante ${ }^{1}$, \\ Helinando Pequeno de Oliveira ${ }^{1}$
}

\footnotetext{
${ }^{1}$ Laboratório de Espectroscopia de Impedância e Materiais Orgânicos, Instituto de Pesquisa em Ciência dos Materiais, Universidade Federal do Vale do São Francisco, Av. Antônio Carlos Magalhães, 510,CEP: 48.902-300, Country Club, Juazeiro, Bahia, Brasil.

e-mail: helinando.oliveira@univasf.edu.br
}

\begin{abstract}
RESUMO
A atividade antibacteriana de nanopartículas metálicas (e em especial de prata) vem sendo explorada ao longo do tempo em diferentes formulações químicas para agentes biológicos livres de antibióticos. A produção dessas partículas por rotas ambientalmente responsáveis representa uma estratégia extremamente relevante por apresentar baixo custo, favorecer a escalabilidade e ter mínimos impactos ambientais. O uso de óleos essenciais como agentes redutores em síntese verde de nanopartículas de prata introduz a sinergia de um agente intrinsicamente antibacteriano e que favorece a nucleação/crescimento das nanopartículas. Esta revisão apresenta o estado da arte da síntese verde de nanopartículas de prata por óleos essenciais assim como discute os mecanismos mais relevantes desse processo de formação e ação antibacteriana.
\end{abstract}

Palavras-chave: Microrganismos. Nanopartícula. Patógeno. Biossíntese.

\section{ABSTRACT}

The antibacterial activity of silver nanoparticles has been explored over time in different chemical formulations for antibiotic-free agents. The production of these particles by environmentally friendly routes represents an extremely relevant strategy for presenting low cost, favoring scalability and having minimal environmental impacts. The use of essential oils as reducing agents in green synthesis of silver nanoparticles introduces the synergy of an intrinsically antibacterial agent that favors the nucleation / growth of the nanoparticles. This review presents the state-of-the-art of green synthesis of silver nanoparticles by essential oils as well as discusses the most relevant mechanisms of formation process and antibacterial action.

Keywords: Microorganisms. Nanoparticles. Pathogen. Biosynthesis.

\section{INTRODUÇÃO}

As nanopartículas metálicas (NPMs) têm recebido atenção crescente na literatura devido às suas propriedades químicas e físicas [1], que conferem atividade antibacteriana [2], catalítica, óptica, eletrônica e magnética [3]. Nanopartículas são estruturas com tamanho entre 1 e 100 nanômetros, caracterizados por densidade local de estados, confinamento quântico e excitação de plásmons de superfície favoráveis a aplicações diversas [4], [5]. A eficiência das NPMs para uso em aplicações tais como citadas acima é favorecida pela maior área superficial , o que confere possibilidade de funcionalização de sítios superficiais ativos, estabilidade e boa capacidade de adsorção [6].

Diferentes NPMs têm sido sintetizada e aplicada em diversas áreas [7], NPMs de ouro e prata [4, 8], além das nanopartículas de zinco, paládio [9] e platina [10], as quais são aplicadas em setores como energia, medicina, agricultura e biotecnologia.

As NPMs são preparadas por três métodos diferentes: químicos, físicos e biológicos. A síntese química ocorre em meio alcoólico, microemulsões, via desintegração térmica de sais metálicos e sínteses eletroquímicas [7]. O método químico envolve a utilização de reagentes químicos geralmente tóxicos e danosos ao meio ambiente e o ser humano [11].

O método físico faz uso de processos de evaporação-condensação, cristalização amorfa, pirólise e mo- 
agem de esferas de alta energia, fragmentação física entre outros processos que envolvem forças de atração entre partículas em escala nanométrica [12], o que ser considerado um limitante, dadas as técnicas de alto custo, envolvendo tratamentos diversos [13].

Já o método biológico se dá pela redução de íons metálicos sob ação de extratos de plantas, óleos essenciais, microrganismos, incluindo bactérias, fungos, leveduras e algas [3, 14, 15]. Esse método é conhecido como síntese verde ou biossíntese e tem recebido destaque na nanotecnologia, por reduzir os impactos ambientais durante a produção de nanopartículas, uma vez que há redução na quantidade de reagentes químicos usados, contribuindo com a geração de subprodutos tóxicos, ou necessidade de equipamentos ou processos dispendiosos [8].

\section{SÍNTESE VERDE OU BIOSSÍNTESE}

A biossíntese, fitossíntese ou síntese verde de metais apresenta como vantagens o consumo reduzido de energia e de insumos químicos sintéticos, a não-necessidade de instalações laboratoriais sofisticadas e solventes tóxicos $[16,17]$.

$\mathrm{Na}$ síntese verde à base de extratos de plantas é possível produzir nanopartículas de prata pela ação dos metabólitos secundários presentes nesses compostos, como flavonoides, terpenos, alcaloides, fenólicos, sacarídeos, entre outras biomoléculas que são capazes de reduzir íons $\mathrm{Ag}^{+} \mathrm{em}$ íons prata metálico $\mathrm{Ag}^{0}$ [18]. Esses extratos vegetais funcionam tanto como agentes redutores como estabilizantes [19].

YOUSAF et al. [20] descrevem a síntese de nanopartículas de prata (AgNPs) utilizando o extrato aquoso da massa seca da planta Achillea millefolium, obtendo NPs com tamanhos entre 14,27 - 20,77 nm, com formas esféricas, retangulares e cúbicas. IZADIYAN et al. [21] aplicaram o extrato aquoso da casca verde da planta Juglans regia na síntese de nanopartículas de óxido de ferro, obtendo nanopartículas com diâmetro médio entre 1,66 - 12,60 nm, com alto grau de pureza e estabilidade.

Microrganismos como bactérias e fungos são também descritos como potenciais candidatos para a síntese de AgNPs com diferentes tamanhos. QUINTEROS et al. [22] utilizaram uma estirpe bacteriana de Pseudomonas aeruginosa para sintetizar AgNPs com tamanhos entre 25 - $45 \mathrm{~nm}$. SRIVASTAVA et al. [8] produziram AgNPs com tamanhos entre 1 a $50 \mathrm{~nm}$, utilizando a espécie de fungo Fusarium oxysporum. Este processo é possível porque fungos e bactérias de diferentes espécies são capazes de excretar enzimas e outras proteínas que funcionam como agentes redutores [3,23]. Ainda há possibilidade de utilizar a própria biomassa morta desses organismos na síntese de NPs por meio de bioprocessos distintos [24]. Entretanto, a síntese de nanopartículas utilizando microrganismos é susceptível a contaminação de culturas, complexidade de procedimentos e um menor controle sobre o tamanho das nanopartículas [16].

Por outro lado, diferentes linhagens de algas e microalgas são utilizadas para produzir nanopartículas metálicas [25, 26]. ÖZTÜRK et al. [27], sintetizaram nanopartículas de prata utilizando pó de algas marinhas vermelhas Gelidium corneum. ANNAMALAI e NALLAMUTHU [25] utilizaram o extrato aquoso da alga Chlorella vulgaris como agente redutor de AgNPs, obtendo nanopartículas entre $15 \mathrm{~nm}$ e $47 \mathrm{~nm}$.

É importante ressaltar que o controle no tamanho nas partículas de agentes antibacterianos, em especial AgNPs é determinante para a toxicidade do produto final. Kim et al. [28] reportaram propriedades antibacterianas como sendo dependentes do tamanho das partículas sintetizadas. AgNPs de $10 \mathrm{~nm}$ demonstraram capacidade de provocar apoptose celular em maior nível que partículas de AgNPs de 50 e $100 \mathrm{~nm}$.

\subsection{Biossínteses mediadas por óleos essenciais}

Além das possibilidades anteriormente descritas, estudos recentes têm avaliado a eficiência dos óleos essenciais de diferentes espécies de plantas na síntese verde de NPMs, visto que esses óleos são substâncias complexas com considerável ação biorredutora. Em pesquisa publicada por ALFURAYDI et al. [29] foi possível sintetizar nanopartículas de prata pela incubação do $\mathrm{AgNO}_{3}$ com o óleo essencial de gergelim (Sesamum indicum), obtendo nanopartículas esféricas com diâmetros entre 6,6 nm e 14,8 nm. Em estudo realizado por DZIMITROWICZ et al. [19] nanopartículas de ouro foram biossintetizadas por meio do contato do óleo essencial de Eucalyptus globulus com a solução aquosa de $\mathrm{HAuCl}_{4}$, obtendo AuNPs esféricas e com tamanho médio de $42 \mathrm{~nm}$.

Os óleos essenciais são utilizados para reduzir íons metálicos em nanopartículas devido a sua complexa diversidade de biomoléculas redutoras. Alguns grupos funcionais dos óleos podem interagir com diferentes íons, formando ligações que favorecem o processo de nucleação de NPs. MELO et al. [30] explicaram o mecanismo de biossíntese de AgNPs com óleo essencial de tomilho (Thymus vulgaris). Segundo os autores, a reação de redução de $\mathrm{Ag}^{+}$em $\mathrm{Ag}^{0}$ ocorreu pela doação de prótons $\mathrm{H}^{+}$do borneol (composto de maior concen- 
tração encontrado no óleo essencial), com a estabilização simultânea do borneol pelo sódio $\left(\mathrm{Na}^{+}\right)$da solução percursora. Na biossíntese de AuNPs com óleo essencial de Ferula persica, HOSSEINZADEH et al. [31], observaram que a presença de ligações tiol em compostos contendo enxofre poderia ser considerado como o responsável por reduzir e revestir os íons de ouro.

A ação redutora do óleo essencial de tomilho (Thymus vulgaris) foi aplicada na síntese de AgNPs, que foi avaliada por sua atividade antibacteriana contra Escherichia coli e Staphylococcus aureus [30]. As nanopartículas apresentaram formato esférico com tamanho médio de $90 \mathrm{~nm}$, e pico de absorbância entre 415 e $440 \mathrm{~nm}$. Dezessete compostos diferentes foram identificados no óleo essencial por cromatografia gasosa, sendo barneol e $\alpha$-terpineol os compostos majoritários, e provavelmente os responsáveis pela formação das AgNPs com potencial atividade antibacteriana.

HOSSEINZADEH et al. [31] sintetizaram nanopartículas de ouro usando óleo essencial de goma de Ferula persica, e investigaram o efeito das nanopartículas produzidas contra o câncer. As nanopartículas de ouro (AuNPs) exibiram forma esférica com diâmetro médio de 37,05 nm. O pico de absorbância na espectroscopia UV-visível foi de $530 \mathrm{~nm}$. E os testes in vitro comprovaram a eficiência da sua atividade anticâncer.

Em outro trabalho foi utilizado o óleo essencial de gengibre (Zingiber zerumbet) para produzir um nanocompósito de óxido de zinco e prata pelo método de síntese verde, com o objetivo de avaliar o efeito bactericida e citotóxico desse nanomaterial. AgNPs foram incorporadas em nanopartículas hexagonais de $\mathrm{ZnO}$. Os testes in vitro confirmaram que a prata melhorou o efeito bactericida das nanopartículas de zinco (ZnO-NPs), demonstrando que o óleo essencial de gengibre foi eficiente no processo de biossíntese, apresentando resultado semelhante a síntese realizada por métodos convencionais, que usam reagentes químicos perigosos [32].

DZIMITROWICZ et al. [19] compararam a eficiência de extratos aquosos de folhas e óleos essenciais de duas espécies de plantas (Eucalyptus globulus e Rosmarinus officinalis) na síntese de nanopartículas de ouro. O extrato aquoso de E. globulus e seu óleo essencial formaram nanopartículas de ouro (AuNPs) com diâmetro médio entre $12,8 \pm 6,3 \mathrm{~nm}$ e 42,2 $\pm 42,0 \mathrm{~nm}$, enquanto o correspondente com a $R$. officinalis, formaram AuNPs com tamanhos entre 8,66 $\pm 2,03 \mathrm{~nm}$ e 60,7 $\pm 60,6$, respectivamente. Ambos os agentes utilizados foram eficientes na produção de AuNPs, contudo os extratos vegetais formaram nanopartículas menores. Possivelmente esse resultado está associado as maiores quantidades de estabilizadores nos extratos brutos do que nos óleos essências.

GA'AL et al. [33] também realizaram uma pesquisa comparando a ação de dois diferentes óleos essenciais (Aquilaria sinensis e Pogostemon cablin) na síntese de nanopartículas de prata, para aplicar ao combate ao mosquito Aedes albopictus. Foram obtidas AgNPs com tamanhos médios variando entre 15 e $87 \mathrm{~nm}$, e as mesmas exibiram ação larvicida significativa. Além disso, as AgNPs apresentaram toxidade contra o mosquito A. albopictus mesmo em doses baixas.

É importante destacar o princípio da reação do óleo essencial na redução da prata. VILAS et al. [34] biossintetizaram AgNPs com óleo essencial de folhas de C. aromaticus, e por cromatografia obtiveram que os compostos em maior concentração foram terpenos e álcoois terpenos. Em temperaturas elevadas e na presença de oxigênio molecular, os hidrocarbonetos terpenos são capazes de clivar ligações duplas, realizar auto-oxidação e desidrogenar. Esse mecanismo leva a formação de radicais livres. Estes radicais livres formados são extremamente eficientes na redução de íons de metais pesados como Ag.

Em geral, a reação de redução da prata envolve o equilíbrio na doação de elétrons. A formação de íons $\mathrm{Ag}^{0}$ e sua sequência de aglomeração é o que se define como nucleação. A união de várias unidades de $\mathrm{Ag}^{0}$ é que forma uma nanopartícula. As biomoléculas dos óleos essências além de participarem da bioredução do $\mathrm{AgNO}_{3}$, podem se organizar na superfície das NPs tornando-as mais estáveis [30].

A Tabela 1 resume os principais produtos obtidos da ação redutora dos óleos em nanopartículas metálicas.

Tabela 1: Nanopartículas metálicas biossintetizadas com óleos essenciais de diferentes espécies de planta.

\begin{tabular}{l|c|c|c|c|c}
\hline \multicolumn{1}{|c|}{ ESPÉCIE DE PLANTA } & NPs & $\begin{array}{c}\text { TAMANHO } \\
(\mathbf{n m})\end{array}$ & FORMA & $\begin{array}{c}\text { ABSORBÂNCIA } \\
(\mathbf{n m})\end{array}$ & Referências \\
\hline Anacardium occidentale & $\mathrm{Au}$ & 36 & Hexagonal & $545-560$ & {$[35]$} \\
\hline Aquilaria sinensis & $\mathrm{Ag}$ & $15-55$ & Esférica & 408 & {$[33]$} \\
\hline Coleus aromaticus & $\begin{array}{c}\mathrm{Au} \mathrm{e} \\
\mathrm{Au} / \mathrm{Ag}\end{array}$ & $14,17 \mathrm{e} 20$ & $\begin{array}{c}\text { Irregular, } \\
\text { anisotrópica }\end{array}$ & $\begin{array}{c}426(\mathrm{Au}) \\
408-526(\mathrm{Au} / \mathrm{Ag})\end{array}$ & {$[36]$} \\
\hline
\end{tabular}




\begin{tabular}{|c|c|c|c|c|c|}
\hline Coleus aromaticus & $\mathrm{Ag}$ & 26 e 28 & Esférica & $396-411$ & [34] \\
\hline Curcuma pseudomontana & $\mathrm{Au}$ & 20 & Esférica & 525 & [37] \\
\hline Curcuma zedoaria & $\mathrm{Ag}$ & - & Globular & 415 & {$[38]$} \\
\hline Eucalyptus globulus & $\mathrm{Au}$ & 42,2 & Esférica & 544,2 & [19] \\
\hline Ferula persica & $\mathrm{Au}$ & 37,05 & Esférica & 530 & [31] \\
\hline Myristica fragrans & $\mathrm{Ag}$ & $12-26$ & Esférica & 420 & [39] \\
\hline Nigella sativa & $\mathrm{Au}$ & 15,6 e 28,4 & Esférica & 540 & [40] \\
\hline Orange peel & $\mathrm{Ag}$ & $6,6-14,80$ & Esférica & 412 & [41] \\
\hline Pogostemon cablin & $\mathrm{Ag}$ & $16-87$ & Esférica & 430 & [33] \\
\hline Rosmarinus officinalis & $\mathrm{Ag}$ & 52 & - & 450 & [42] \\
\hline Rosmarinus officinalis & $\mathrm{Au}$ & $60,7 \pm 60,6$ & Esférica & 528,9 & [19] \\
\hline Sesamum indicum & $\mathrm{Ag}$ & 6,6 e 14,8 & Esférica & 420 & [29] \\
\hline Syzygium aromaticum & $\mathrm{Ag}$ & $31-72$ & Esférica & 430 & [43] \\
\hline Thymus vulgaris & $\mathrm{Ag}$ & 40 & Esférica & $415-440$ & [30] \\
\hline Zingiber zerumbet & $\mathrm{ZnO}$ & 5,23 e 45 & Hexagonal & 430 & [32] \\
\hline
\end{tabular}

2.2 Mecanismos gerais para o processo de redução de nanopartículas induzido pelos óleos essenciais

Óleos essenciais favorecem a síntese verde de nanopartículas metálicas e envolvem três possíveis reações de complexação. A primeira reação ocorre entre os componentes principais dos óleos essenciais, como: ácidos fenólicos, flavonóides, terpenóides e antocianinas; e os íons metálicos [44].

A segunda reação possível se dá entre os grupos hidroxila de antocianinas, flavonóides e ácidos fenólicos dos óleos essenciais podendo conduzir diretamente à redução dos íons metálicos. Assim, as substâncias principais dos óleos, por meio de seus grupos hidroxila, combinam-se com os íons metálicos resultando na formação de alguns compostos antes de produzir as nanopartículas metálicas finais por meio da redução [45].

Esses compostos de transição formam as NPs por transferência de elétrons, as nanopartículas passam a ser revestidas por antocianinas e ácidos fenólicos.

Na terceira reação, que é muito mais lenta, as outras biomoléculas presentes no óleo essencial, que não apresentam capacidade redutora na solução, difundem continuamente para as interfaces sólido-líquido formadas no núcleo das nanopartículas metálicas [46], com esquema de complexação apresentado na Figura 1. 


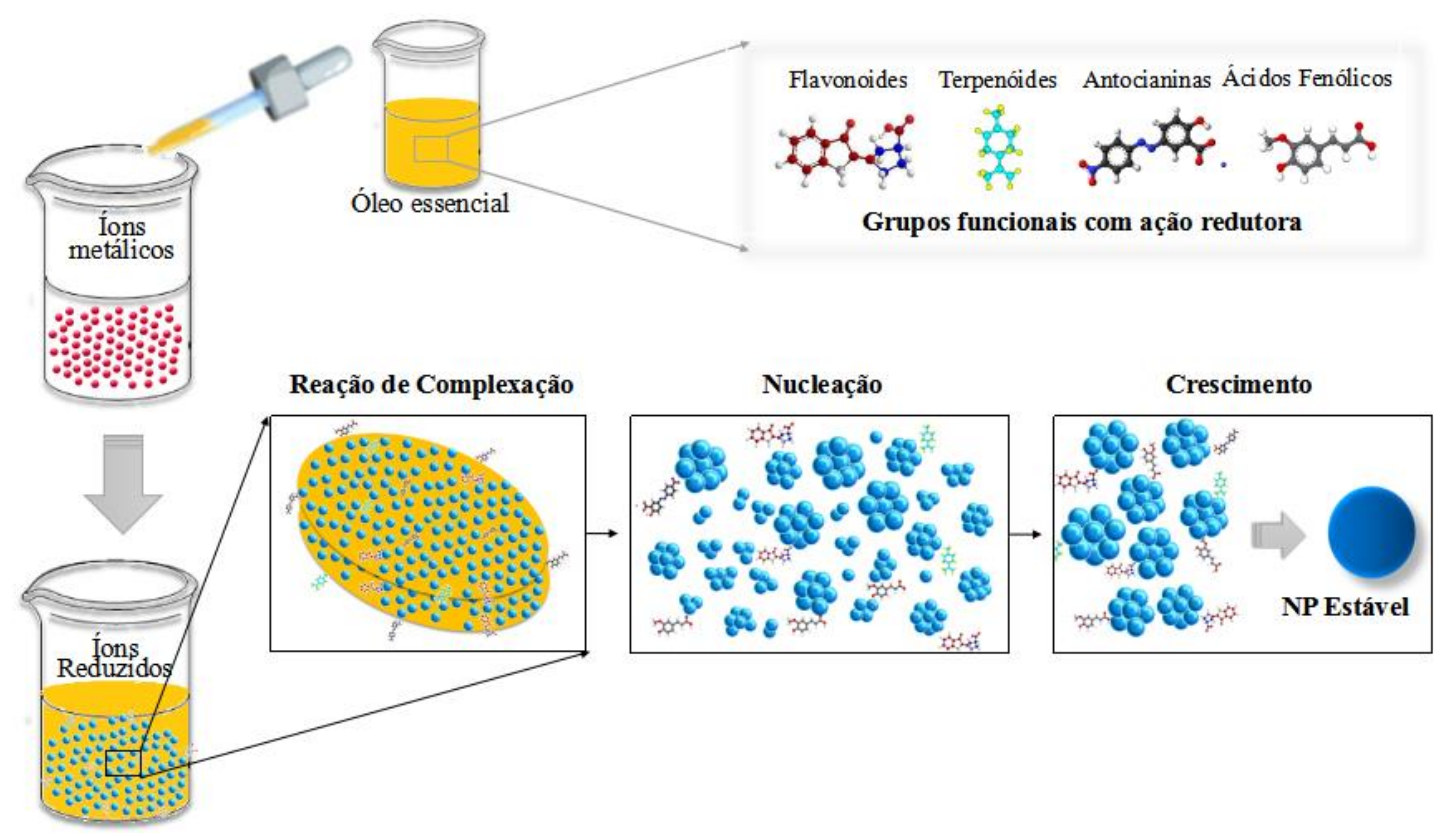

Figura 1: Reação de complexação, como possível mecanismo da síntese verde de nanopartículas metálicas mediado por óleos essenciais.

O revestimento de superfície não redutora derivado do óleo essencial pode ser absorvido pelas nanopartículas metálicas, contribuindo com a maior estabilidade nas partículas sintetizadas [46].

Com isso o processo de formação de nanopartículas pode ser generalizado por três etapas principais: a oxirredução, nucleação e crescimento [32, 34], como descrito na Figura 2.

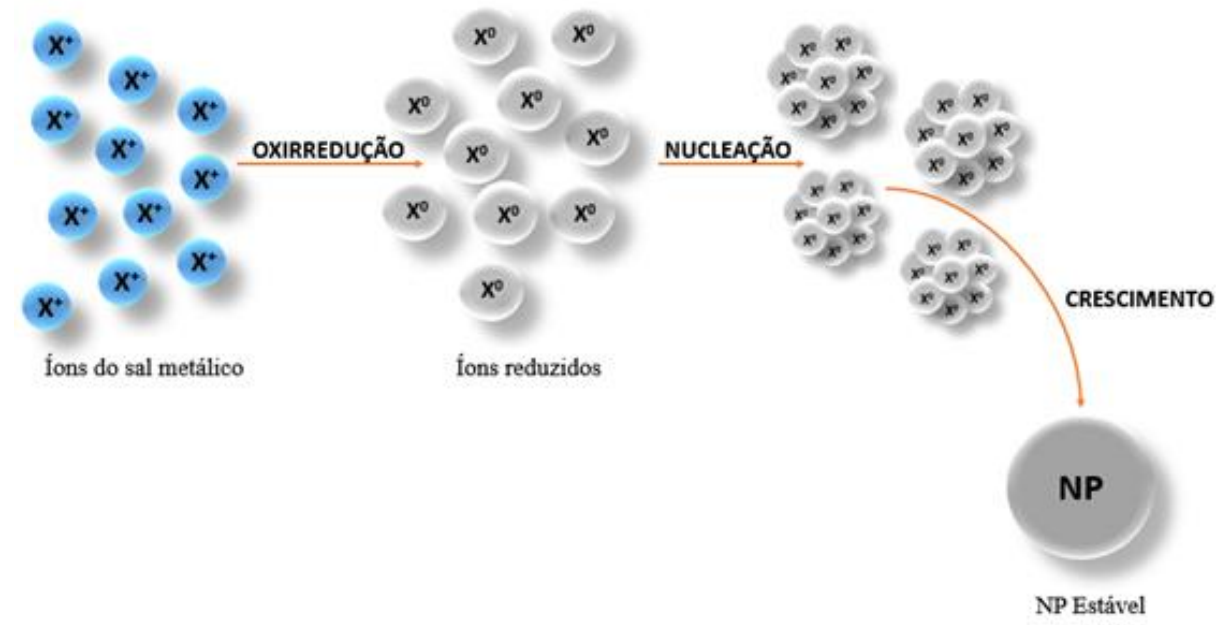

Figura 2: Esquema geral de preparação de NPs

Na etapa de oxirredução os grupos funcionais dos óleos essenciais (agente redutor) contendo oxigênio no ligante doam elétrons $\pi$ para o metal de transição (agente oxidante). Neste processo, o íon metálico sofre redução à medida em que oxida os componentes químicos do óleo essencial. Em alguns casos, ocorre a decomposição térmica do complexo iônico do sal metálico percursor aumentando a velocidade da reação [47].

Nas etapas de nucleação e crescimento, há o desencadeamento de uma série de fases metaestáveis promovida pela ação dos óleos essenciais, até a solução coloidal atingir um estágio termodinamicamente estável [48].

Com a estabilidade termodinâmica, os átomos que sofreram redução no meio reacional, dão origem a grupos que ao atingir determinado tamanho característico formam um núcleo estável. O menor raio para formação de um núcleo estável é caracterizado pelo seu raio crítico, cujas características são dadas pela capaci- 
dade do óleo de manter a estabilidade da energia livre de superfície em relação a área superficial do grupo de nucleação. Dessa forma, quanto maior o equilíbrio entre esses dois fatores, maior o tamanho do núcleo crítico estável [49].

Relativamente aos estudos de nucleação, há dois tipos de processos: a nucleação homogênea e a nucleação heterogênea. A nucleação homogênea ocorre em uma única etapa, onde a concentração atinge uma condição de supersaturação crítica, e permite crescimento uniforme por difusão dos átomos reduzidos para o núcleo formado. Já na nucleação heterogênea, a formação de núcleos ocorrer pela ação de agentes nucleantes, sendo estes os promotores do crescimento das partículas [50].

A etapa seguinte a nucleação é denominada de crescimento, processo em que os núcleos migram para a superfície do soluto. Nesse processo ocorre o transporte de espécies reativas para a superfície das partículas, coalescência e agregação que podem competir com o processo de crescimento [51].

Nesse ponto a ação estabilizante do óleo pode equilibrar o crescimento da nanopartícula pela estabilização eletrostática da dupla camada elétrica na superfície das NPs (repulsão coulombiana), diminuindo o índice de polidispersividade das nanopartículas (PDI) [51].

\subsection{Fatores que influenciam a biossíntese de nanopartículas metálicas}

Diferentes fatores estão envolvidos no processo de síntese de nanopartículas metálicas. Uma quantidade significativa de trabalhos vem estudando a ação e a interferência de parâmetros químicos e físicos envolvidos na redução de íons metálicos $[32,19]$.

A temperatura, o pH e o tempo de reação são os fatores que mais interferem nas características morfológicas das nanopartículas (Tabela 2). Na síntese verde, a concentração e a quantidade do agente redutor biológico também é um dos fatores que atua sobre o tamanho, a forma e a estabilidade das NPs [35]. A combinação entre $\mathrm{pH}$ e temperatura passam a determinar o tamanho e forma dessas nanoestruturas [52] dada a importância do papel da agitação térmica na eficiência do processo de nucleação e crescimento das nanoestruturas. Como descrito, o aumento na temperatura na faixa entre 20 e $60^{\circ} \mathrm{C}$ possibilita um aumento tanto na banda de plásmons (características da formação de nanoestruturas) quanto na taxa de formação das nanopartículas, que passam a ter uma redução em seu tamanho médio, dada a contribuição que a temperatura proporciona na taxa de nucleação das nanoestruturas.

Tabela 2: Parâmetros estabelecidos no processo de biossíntese de nanopartículas metálicas utilizando óleo essencial de diferentes espécies de plantas.

\begin{tabular}{|c|c|c|c|c|c|}
\hline $\begin{array}{l}\text { ESPÉCIE DE } \\
\text { PLANTA }\end{array}$ & NPs & TEMPERATURA & $\mathrm{pH}$ & $\begin{array}{l}\text { TEMPO DE } \\
\text { REAÇÃO }\end{array}$ & REFS. \\
\hline Anacardium occidentale & $\mathrm{Au}$ & $100^{\circ} \mathrm{C}$ & - & $1 \mathrm{~min}$ & {$[35]$} \\
\hline Aquilaria sinensis & $\mathrm{Ag}$ & - & 7 & $90 \mathrm{~min}$ & {$[33]$} \\
\hline Coleus aromaticus & $\mathrm{Au}$ e $\mathrm{Au} / \mathrm{Ag}$ & - & 7 & - & {$[36]$} \\
\hline Coleus aromaticus & $\mathrm{Ag}$ & - & 7 & - & {$[34]$} \\
\hline $\begin{array}{l}\text { Curcuma pseudomonta- } \\
\text { na }\end{array}$ & $\mathrm{Au}$ & $95^{\circ} \mathrm{C}$ & - & $30 \mathrm{~min}$ & {$[37]$} \\
\hline Curcuma zedoaria & $\mathrm{Ag}$ & - & 7 & $180-240 \mathrm{~min}$ & {$[38]$} \\
\hline Eucalyptus globulus & $\mathrm{Au}$ & $95^{\circ} \mathrm{C}$ & - & - & [19] \\
\hline Ferula persica & $\mathrm{Au}$ & Ambiente & - & $1440 \mathrm{~min}$ & {$[31]$} \\
\hline Myristica fragrans & $\mathrm{Ag}$ & $100^{\circ} \mathrm{C}$ & 7 & - & [39] \\
\hline Nigella sativa & $\mathrm{Au}$ & $100^{\circ} \mathrm{C}$ & - & $1 \mathrm{~min}$ & {$[40]$} \\
\hline Orange peel & $\mathrm{Ag}$ & $80^{\circ} \mathrm{C}$ & & $480 \mathrm{~min}$ & [41] \\
\hline
\end{tabular}




\begin{tabular}{l|c|c|c|c|c}
\hline Pogostemon cablin & $\mathrm{Ag}$ & - & 7 & - & [33] \\
\hline Rosmarinus officinalis & $\mathrm{Ag}$ & $80^{\circ} \mathrm{C}$ & - & $25 \mathrm{~min}$ & {$[42]$} \\
\hline Rosmarinus officinalis & $\mathrm{Au}$ & $95^{\circ} \mathrm{C}$ & - & - & {$[19]$} \\
\hline Sesamum indicum & $\mathrm{Ag}$ & $\mathrm{Ambeinte}$ & - & $2880 \mathrm{~min}$ & {$[29]$} \\
\hline Syzygium aromaticum & $\mathrm{Ag}$ & $100^{\circ} \mathrm{C}$ & $7,8,9 \mathrm{e} 10$ & $30 \mathrm{~min}$ & {$[43]$} \\
\hline Thymus vulgaris & $\mathrm{Ag}$ & $100^{\circ} \mathrm{C}$ & $7,8,9 \mathrm{e} 10$ & $30 \mathrm{~min}$ & {$[30]$} \\
\hline Zingiber zerumbet & $\mathrm{ZnO}$ & $80^{\circ} \mathrm{C}$ & - & $180 \mathrm{~nm}$ & {$[32]$} \\
\hline
\end{tabular}

NISHANTHI et al. [53] sintetizaram nanopartículas de prata (AgNP), ouro (AuNP) e platina (PtNP) usando o extrato da casca do fruto de Garcinia mangostana. A formação de AuNPs ocorreu rapidamente à temperatura ambiente $\left(30-35^{\circ} \mathrm{C}\right)$, enquanto as AgNPs e PtNPs só ocorreram a uma temperatura de $80{ }^{\circ} \mathrm{C}$. Esse processo ocorre pela reação entre o aumento da temperatura e o aumento na taxa de reação e formação de centros de nucleação [52].

$\mathrm{O}$ pH do meio reacional também fornece um papel fundamental na formação de NPs. Assim como a temperatura, o pH regula a formação de centros de nucleação. À medida que o pH aumenta, o número de centros de nucleação também aumentam [53]. É importante ressaltar neste ponto que a oxidação de grupos fenólicos é acompanhada pela liberação de íons $\mathrm{H}^{+}$, e por isso é esperado que a nucleação das AgNPs seja favorecido em meio alcalino, o que leva à síntese de partículas menores e também pela produção de $\mathrm{Ag}_{2} \mathrm{O}$, de acordo com a reação:

$$
2 \mathrm{Ag}^{+}+2 \mathrm{OH}^{-} \rightarrow \mathrm{Ag}_{2} \mathrm{O}+\mathrm{H}_{2} \mathrm{O}
$$

Em estudo realizado por MACIEL et al. [43] nanopartículas de prata (AgNPs) foram sintetizadas pela ação do óleo essencial de cravo-da-índia (Syzygium aromaticum), avaliando a formação das nanopartículas

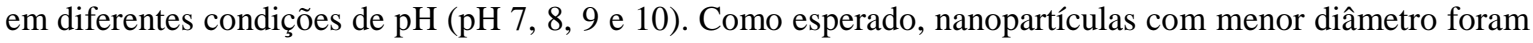
formadas com o aumento no $\mathrm{pH}$ da solução, sendo também observado um aumento no índice de polidispersidade das mesmas e do potencial zeta para $\mathrm{pH}$ variando de 7 a 10 (ordem crescente). As AgNPs apresentaram tamanhos entre 31 e $72 \mathrm{~nm}$, e diferentes formas (triangulares, quadradas, hexagonais e em maioria esferas), dependendo do $\mathrm{pH}$.

Na pesquisa realizada com óleo essencial de tomilho para síntese de AgNPs, MELO et al. [30] também avaliaram a formação das NPs em diferentes condições de $\mathrm{pH}(\mathrm{pH} 7,8,9$, e 10) (Tabela 4). Entretanto a maior formação de AgNPs ocorreu em pH 10 (absorbância máxima em $440 \mathrm{~nm}$ ). Em relação ao tamanho das AgNPs, o pH da solução interferiu diferentemente no diâmetro médio das AgNPs. Em pH 7 houve formação de NPs com $40 \mathrm{~nm}$ de diâmetro, já em pH 10 as AgNPs formadas apresentaram maior tamanho $(90 \mathrm{~nm})$.

$\mathrm{O}$ pH também afeta criticamente a cinética de nucleação de nanopartículas de prata. Utilizando extrato foliar de Ziziphus joazeiro, GUIMARÃES et al. [54] produziram AgNPs com diferentes tamanhos, distribuição e nível de agregação de acordo com o pH do meio reacional. Em ambiente alcalino (pH 11) as AgNPs apresentaram alto índice de polidispersividade (PDI) com NPs de diferentes tamanhos. Em condição neutra $(\mathrm{pH} 7)$, foram obtidas partículas com menor tamanho e grau de agregação reduzido.

Juntamente com a temperatura e o $\mathrm{pH}$, o tempo de reação também é descrito como um dos principais fatores que influenciam a morfologia das nanoparticulas, uma vez que a cinética prolongada de reação pode ser conduzida para um processo de agregação excessiva dos núcleos formadores de nanoestruturas - produzindo agregados de prata em escala microscópica. A variação nas condições do meio $(\mathrm{pH})$ e da cinética de reação que é favorecida pela energia térmica fornecida ao sistema determinam um tempo característico para que a reação seja otimizada, produzindo materiais com índice polidispersão baixo. Para além destas condições (reação prolongada - para além do tempo "ótimo") é induzida a formação de grandes agregados, o que afeta a propriedade antibacteriana do sistema.

VEISI et al. [41] sintetizaram AgNPs com óleo essencial de casca de laranja. As soluções preparadas foram monitoradas em diferentes intervalos de tempo $(0 \mathrm{~h}, 12 \mathrm{~h}, 14 \mathrm{~h}, 36 \mathrm{~h}$ e $48 \mathrm{~h})$ a uma temperatura de $70{ }^{\circ} \mathrm{C}$. No espectro UV-vis, a absorbância das AgNPs aumentaram com o período de interação do nitrato de prata com o óleo essencial. O tempo de reação mostrou-se importante na redução do íon $\mathrm{Ag}^{+} \mathrm{em}^{\mathrm{Ag}}$, sendo a 
maior absorbância (412 nm) encontrada após 48 horas de reação.

Em trabalho com o óleo essencial de gergelim (Sesamum indicum) aplicado à síntese de nanopartículas de prata, foi monitorado a formação das AgNPs em diferentes intervalos de tempo (96, 120, 146 e $170 \mathrm{~h}$ ). A mudança de cor da solução de incolor para marrom ocorreu após 48 h, confirmando formação de AgNPs. A partir do espectro UV-Vis foi obtido banda de Ressonância Plasmônica de Superfície (RPS) característica das AgNPs, e aumento da absorbância com o tempo de interação entre a solução de $\mathrm{AgNO}_{3}$ e o óleo essencial [29].

\subsection{Atividade antibacteriana de NPMs biossintetizadas com óleos essenciais.}

As nanopartículas de ouro [40], óxido de zinco [32] e prata [43], sintetizadas com óleos essenciais têm sido aplicadas como agentes antibacterianos alternativos no combate de bactérias patogênicas. Os principais resultados encontrados na literatura são apresentados na Tabela 3, que mostram a atividade antibacteriana pelo método de halo de inibição onde os resultados (-) indicam resultado não sensível com halo com diâmetro inferior a $8 \mathrm{~mm},(+)$ sensível com halo entre 9-14 mm, (++) muito sensível com halo entre 15-19 mm e (+++) extremamente sensível com halo maior que $20 \mathrm{~mm}$.

Tabela 3: Atividade antibacteriana de nanopartículas metálicas biossíntetizadas com diferentes óleos essenciais.

\begin{tabular}{|c|c|c|c|c|c|}
\hline \multirow{2}{*}{$\begin{array}{c}\text { ESPÉCIE } \\
\text { DE PLANTA } \\
\text { DO OE }\end{array}$} & \multirow[b]{2}{*}{ NPs } & \multicolumn{2}{|c|}{ BACTÉRIA TESTADA } & \multirow[b]{2}{*}{$\begin{array}{c}\text { PRINCIPAIS } \\
\text { RESULTADOS }\end{array}$} & \multirow[b]{2}{*}{ REFS. } \\
\hline & & $\begin{array}{c}\text { GRAM- } \\
\text { NEGATIVA }\end{array}$ & $\begin{array}{c}\text { GRAM- } \\
\text { POSITIVA }\end{array}$ & & \\
\hline $\begin{array}{l}\text { Sesamum indi- } \\
\text { cum }\end{array}$ & $\mathrm{Ag}$ & $\begin{array}{c}\text { P.aeruginosa, } \\
\text { K. pneumoniae, } \\
\text { E.coli }\end{array}$ & $\begin{array}{l}\text { S.aureus, } \\
\text { B.subtilis }\end{array}$ & $\begin{array}{c}\text { P.aeruginosa: - } \\
\text {,K. pneumoniae: - } \\
\text { E.coli: - } \\
\text { S. aureus: + } \\
\text { B. subtilis: + }\end{array}$ & [29] \\
\hline $\begin{array}{l}\text { Zingiber zerum- } \\
\text { bet }\end{array}$ & $\mathrm{ZnO}$ & $\begin{array}{l}\text { S.choleraesuis } \\
\text { E. coli, } \\
\text { A. anitratus }\end{array}$ & $\begin{array}{l}\text { S. aureus, } \\
\text { B. subtilis. } \\
\text { S. epidermidis }\end{array}$ & $\begin{array}{c}\text { E. coli: }++ \\
\text { B. subtilis: ++ } \\
\text { S.choleraesuis: - } \\
\text { A. anitratus: - } \\
\text { S. aureus: - } \\
\text { S. epidermidis: - }\end{array}$ & {$[32]$} \\
\hline $\begin{array}{l}\text { Syzygium aro- } \\
\text { maticum }\end{array}$ & $\mathrm{Ag}$ & E. coli & $\begin{array}{l}\text { S. aureus, } \\
\text { B. cereus }\end{array}$ & $\begin{array}{c}\text { E. coli: }++ \\
\text { S. aureus: }++ \\
\text { B. cereus: - }\end{array}$ & [43] \\
\hline Nigella sativa & $\mathrm{Au}$ & V. harveyi & S. aureus & $\begin{array}{l}\text { S. aureus: }+++ \\
\text { V. heryeyi: ++ }\end{array}$ & [40] \\
\hline Thymus vulgaris & $\mathrm{Ag}$ & E. coli & $\begin{array}{l}\text { S. aureus, } \\
\text { B. cereus }\end{array}$ & $\begin{array}{l}\text { E. coli: }++ \\
\text { S. aureus: +++ } \\
\text { B. cereus: - }\end{array}$ & {$[30]$} \\
\hline $\begin{array}{l}\text { Coleus aromati- } \\
\text { cus }\end{array}$ & $\begin{array}{c}\mathrm{Au} \mathrm{e} \\
\mathrm{Au} / \mathrm{Ag}\end{array}$ & E. coli & S. aureus & $\begin{array}{l}\text { E. coli: }++ \\
\text { S. aureus: }++\end{array}$ & {$[36]$} \\
\hline $\begin{array}{l}\text { Myristica fra- } \\
\text { grans }\end{array}$ & $\mathrm{Ag}$ & E. coli & S. aureus & $\begin{array}{c}\text { E. coli: }+++ \\
\text { S. aureus: ++ }\end{array}$ & [39] \\
\hline
\end{tabular}

OE, Óleo essencial

É importante ressaltar que a medida do halo de inibição apresentado na Tabela 3 representa uma estimativa qualitativa da ação antibacteriana dos compostos, caracterizando a capacidade de difusão das espécies com elevada toxicidade frente às bactérias. Como podemos perceber, não há uma relação direta entre o óleo essencial e o tamanho do halo de inibição, uma vez que a ação antibacteriana das nanopartículas sintetizadas depende de múltiplos fatores, como por exemplo, a interação das nanopartículas com as paredes celulares que variam de acordo com a natureza da bactéria (gram-positiva ou gram-negativa) e às próprias características de cada organismo. No entanto, o aspecto mais importante se refere à atividade antibacteriana de todas as nanopartículas sintetizadas, demonstrando o potencial da síntese verde de nanopartículas antibacterianas. 
Um desafio de praticamente todos estes trabalhos de pesquisa tem sido entender quais os principais mecanismos de ação das NPs no combate de bactérias. VILAS et al. [36] observaram como as diferenças na parede celular de bactérias Gram-negativas e Gram-positivas interferiram na ação das NPs de Au/Ag biossintetizadas com óleo essencial de Coleus aromaticus. As NPs foram mais eficientes no controle do crescimento bacteriano da E. coli (Gram-negativa) do que da bactéria S. aureus (Gram-positiva), que possui uma estrutura capaz de restringir a ação e a permeabilidade das NPs nas células, chamada de peptidoglicano.

Resultado semelhante foi encontrado por ALFURAYDI et al. [29] em pesquisa com AgNPs sintetizadas com óleo essencial de Sesamum indicum. As AgNPs inibiram o crescimento de bactérias Gram-negativas (P. aeruginosa, K. pneumoniae e E. coli), mas não foram eficientes no combate de bactérias Gram-positivas (B. subtilis e $S$. aureus). Esse resultado pode ser associado a capacidade das AgNPs em se ligar à superfície da membrana celular das bactérias Gram-negativas, causando alteração na respiração e permeabilidade celular, além da barreira biológica de proteção encontrada nas bactérias Gram-positivas (camada de peptídoglicano), que dificulta a ação das NPs [29].

Em contrapartida, MANJU et al. [40] avaliaram a ação de AuNPs biossintetizadas com óleo essencial de Nigella sativa, obtendo maior atividade contra a bactéria S. aureus (Gram-positiva), com halos de inibição de $16 \mathrm{~mm}$, do que contra Vibrio harveyi (Gram-negativa), com halos de inibição de $5 \mathrm{~mm}$.

$\mathrm{O}$ tamanho e a forma das nanopartículas metálicas interferem diretamente na sua atividade antibacteriana. A alta relação superfície/volume de NPs aumenta sua afinidade com a membrana celular de bactérias. Além disso, a atração entre cargas positivas e negativas das nanopartículas e membrana celular bacteriana, respectivamente, aumenta devido à presença de lipopolissacarídeo ou ácido tecóico [55].

Em estudo conduzido por MELO et al. [30] nanopartículas de prata com diâmetros médios de $40 \mathrm{~nm}$ e predominância de forma esférica foram mais eficientes no controle de bactéria Gram-negativa (E. coli) e Gram-positiva (S. aureus) do que AgNPs maiores $(90 \mathrm{~nm})$ e com predominância de forma de hastes. Corroborando com estudo realizado por MACIEL et al. [43] que também discutiram a influência da forma e tamanho das NPs com a capacidade de inibir o crescimento bacteriano.

Nanocompósitos de $\mathrm{ZnO}-\mathrm{Ag}$ e nanopartículas $\mathrm{ZnO}$, apresentaram atividades antibacterianas em relação a Bacillus subtilis e a Escherichia coli. Contudo as atividades antibacterianas das amostras variaram de acordo com o microrganismo. Foi observado que a dopagem de $\mathrm{ZnO}$ com $\mathrm{Ag}$ aumentou a atividade bactericida. AZIZI et al. [32], explicam que o pequeno tamanho dos cristais e a grande área de superfície do ZnOAg podem ter contribuído.

Estudos demostram que as nanopartículas metálicas também são eficientes no combate à formação de biofilme [40]. As NPMs podem prejudicar a síntese de exopolissacarídeos (EPS), impossibilitando que ocorra as interações entre bactérias e células hospedeiras. Com diminuição da síntese de EPS, a hidrofobicidade da superfície celular é reduzida, afetando diretamente a produção de biofilme em diferentes microrganismos [56].

Investigações com AuNPs contra a formação de biofilme bacteriano apresentou resultados favoráveis contra S. aureus e V. harveyi. Dependente da dose, as AuNPs foram capazes de desintegrar e inibir a hidrofobicidade das colônias de $S$. aureus e $V$. harveyi, em $78 \%$ e 46\%, respectivamente. Essa resposta pode estar associada à inibição da síntese de exopolissacarídeos (EPS) [40] confirmando a eficiência de NPs como agentes inibidores de biofilme bacteriano.

A produção das nanopartículas metálicas como agentes antibacterianos é uma tecnologia bem fundamentada na literatura, principalmente com as nanopartículas de $\mathrm{Ag}$ e Au. A influência dos óleos essenciais no processo de biossíntese de nanopartículas é uma estratégia promissora tanto do ponto de vista de morfologia quanto do papel antibacteriano para o sistema resultante.

\section{CONCLUSÕES}

Além de funcionar como excelentes protótipos de materiais in natura com potencial antibacteriano, ao apresentar resultados muito expressivos frente a cepas de patógenos, os óleos essenciais ainda não despertaram a devida atenção da literatura para sua ação na síntese verde de nanopartículas metálicas. Sua utilização em rotas ambientalmente amigáveis reduz o custo de produção ao mesmo tempo em que permite com que a morfologia das partículas sintetizadas seja controlada. Assim, há a possibilidade de controlar a atividade antibacteriana das NPs pelo tamanho e forma dessas nanoestruturas, sendo as nanopartículas de menor tamanho e de formato esférico, as que apresentaram melhores resultados. Outro fator que demanda importante perspectivas de investigação se refere ao sinergismo entre as nanopartículas sintetizadas e o meio reacional. O sinergismo do meio reacional utilizado para reduzir as nanopartículas de prata e a própria ação inerente destas é um importante tópico a ser explorado em trabalhos futuros. 


\section{AGRADECIMENTOS}

Agradecemos à CAPES, ao CNPq e à FACEPE pelo apoio ao desenvolvimento de pesquisa no LEIMOIPCM/ Univasf.

\section{BIBLIOGRAFIA}

[1] OVAIS, M., KHALIL, A.T., AYAZ, M., et al., "Biosynthesis of Metal Nanoparticles via Microbial Enzymes: A Mechanistic Approach" Internation Journal of Molecular Sciences, v. 19, n. 12 pp. 1-20, Dec. 2018.

[2] PANIZ, O.G., SCHEIK, L.K., SILVA, G.E.H., et al., "Cellulose acetate and silver particles composite synthesis to antimicrobial applications" Revista Materia, vol. 23, no. 4, pp. e12244, Dec. 2018.

[3] NASROLLAHZADEH,, M., SAJADI, S.M., SAJJADI, M., et al., "An Introduction to Nanotechnology" in An Introduction to Green Nanotechnology, vol. 28, pp. 1-27, 2019.

[4] N. NOAH, "Green synthesis: Characterization and application of silver and gold nanoparticles" in Green Synthesis, Characterization and Applications of Nanoparticles, Elsevier Inc., pp. 111-135, 2019.

[5] RODRIGUES, J. F. B., BRANDÃO, P.E.S., GUIMARÃES, P.Q., et al., "Application of statistical method in the study of the influence of hydrogen peroxide and sodium borohydride on silver nanoparticle synthesis (AGNPS)" Revista Materia, v. 24, n. 3, pp. e-12395, Feb. 2019.

[6] RAI, P.K., KUMAR, V., LEE, S., et al., "Nanoparticle-plant interaction: Implications in energy , environment, and agriculture" Environment International, v. 119, n. April, pp. 1-19, 2018.

[7] TULINSKI, M., JURCZYK, M., "Nanomaterials Synthesis Methods" Metrology and Standardization for Nanotechnology, n. 1, pp. 75-98, Jan. 2017.

[8] SRIVASTAVA, S., BHARGAVA, A., PATHAK, N., et al., "Production, characterization and antibacterial activity of silver nanoparticles produced by Fusarium oxysporum and monitoring of proteinligand interaction through in-silico approaches" Microbial Pathogenesis, v. 129, pp. 136-145, Apr. 2019.

[9] POURREZA N., ABDOLLAHZADEH, R., "Colorimetric sensing of palladium ions based on in situ generation of palladium nanoparticles as an activator for the thionine-hydrazine reaction" Talanta, v. 196, pp. 211-216, May 2019.

[10] GUGGILLA, B.R., RUSTED, A., AND BAKRANIA, S., "Platinum nanoparticle catalysis of methanol for thermoelectric power generation" Applied Energy, v. 237, pp. 155-162, Oct. 2019.

[11] SOLGI, M., TAGHIZADEH, M., "Biogenic Synthesis of Metal Nanoparticles by Plants" Biogenic Nano-Particles and their Use in Agro-ecosystems, pp. 593-606, 2020.

[12] TNVKV, P., AND EK, E., "Biofabrication of Ag nanoparticles using Moringa oleifera leaf extract and their antimicrobial activity" Asian Pacific Journal of Tropical Biomedicine, v. 1, n. 6, pp. 439-442, Dec. 2011.

[13] ABDELGHANY, T.M., AL-RAJHI, A.M.H., AL ABBOUD, M.A., "Recent Advances in Green Synthesis of Silver Nanoparticles and Their Applications: About Future Directions . A Review" BioNanoScience, v. 8, pp. 5-16, Apr. 2018.

[14] AHMAD, S., SIDRA, M., ZEB, N., et al., , "Green nanotechnology: a review on green synthesis of silver nanoparticles - an ecofriendly approach" International journal of nanomedicine, pp. 5087-5107, Jul. 2019.

[15] MAGHIMAA, M., ALI, S., "Biology Green synthesis of silver nanoparticles from Curcuma longa L . and coating on the cotton fabrics for antimicrobial applications and wound healing activity" Journal of Photochemistry \& Photobiology, B: Biology, v. 204, pp. 111806, Mar. 2020.

[16] SIDDIQUE, S., PARVEEN, Z., AND MAZHAR, S., "Chemical composition , antibacterial and antioxidant activities of essential oils from leaves of three Melaleuca species of Pakistani flora" Arabian Journal of Chemistry, v. 13, n. 1, pp. 67-74, Jan. 2017.

[17] CRUZ, N.D., GALLIO, E., AND GATTO, D.A., "Green synthesis of zinc oxide nanoparticles" Revista Materia, v. 25, n. 1, e-12589, Apr. 2020.

[18] BEHRAVAN, M., HOSSEIN, A., NAGHIZADEH, A., et al., "Facile green synthesis of silver nanoparticles using Berberis vulgaris leaf and root aqueous extract and its antibacterial activity" International Journal of Biological Macromolecules, v. 124, pp. 148-154, Mar. 2019.

[19] DZIMITROWICZ, A., BERENT, S., MOTYKA, A., et al., "Comparison of the characteristics of gold nanoparticles synthesized using aqueous plant extracts and natural plant essential oils of Eucalyptus globulus and Rosmarinus officinalis" Arabian Journal of Chemistry, v. 12, n. 8, pp. 4795-4805, Dec. 2019.

[20] YOUSAF, H., MEHMOOD, A., SHAFIQUE, K. et al., "Green synthesis of silver nanoparticles and their 
applications as an alternative antibacterial and antioxidant agents" Materials Science \& Engineering C, v. 112, n. March, p. 110901, 2020.

[21] IZADIYAN, Z.,. SHAMELI, K., MIYAKE, M., et al., "Cytotoxicity assay of plant-mediated synthesized iron oxide nanoparticles using Juglans regia green husk extract" Arabian Journal of Chemistry, v. 13, n. 1, pp. 2011-2023, Dec. 2020.

[22] QUINTEROS, M.A., MARTÍNEZ, I.M.A., DALMASSO, P.R. et al., "Silver Nanoparticles : Biosynthesis Using an ATCC Reference Strain of Pseudomonas aeruginosa and Activity as Broad Spectrum Clinical Antibacterial Agents" International Journal of Biomaterials, v. 2016, May 2016.

[23] VARDHANA, J., AND KATHIRAVAN, G., "Biosynthesis of silver nanoparticles by endophytic fungi Pestaloptiopsis pauciseta isolated from the leaves of Psidium guajava linn" International Journal of Pharmaceutical. Sciences Review and Research, v. 31, n. 1, 29-21, Dec. 2014.

[24] THOMAS, R., JANARDHANAN, A., VARGHESE, et al., "Antibacterial properties of silver nanoparticles synthesized by marine Ochrobactrum sp ." Brazilian Journal of Microbiology, v. 1227, pp. 1221-1227, Dec. 2014.

[25] ANNAMALAI J., NALLAMUTHU, T., "Green synthesis of silver nanoparticles : characterization and determination of antibacterial potency" Applied Nanoscience, pp. 259-265, Mar. 2016.

[26] KHALIFA, K.S. HAMOUDA, R.A., HAMZA, H.A., "Antitumor activity of silver nanoparticles biosynthesized by micro algae" Journal of Chemical and Pharmaceutical Research, v. 8, n. 3, pp. 1-6, Dec. 2016.

[27] ÖZTÜRK, B.Y., GÜRSU, B.Y., DAĞ, İ., “Antibiofilm and antimicrobial activities of green synthesized silver nanoparticles using marine red algae Gelidium corneum" Process Biochemistry, v. 89, n. September 2019, pp. 208-219, 2020.

[28] KIM T.H., KIM M., PARK, H.S., et al., "Size-dependent cellular toxicity of silver nanoparticles" Journal of Biomedical Materials Research - Part A, v. 100 A, n. 4, pp. 1033-1043, Feb. 2012.

[29] ALFURAYDI, A.A., DEVANESAN, S., AL-ANSARI, M. , et al., " Eco-friendly green synthesis of silver nanoparticles from the sesame oil cake and its potential anticancer and antimicrobial activities" Journal of Photochemistry \& Photobiology, B: Biology, v. 192, n. January, pp. 83-89, 2019.

[30] MELO, A.P.Z., O.B. MACIEL, M.V., SGANZERLA, W.G., et al., "Antibacterial activity , morphology , and physicochemical stability of biosynthesized silver nanoparticles using thyme ( Thymus vulgaris) essential oil Antibacterial activity, morphology, and physicochemical stability of biosynthesized silver nanoparti” Materials Research Express, IOP Publishing, p. 015087, Jan. 2020.

[31] HOSSEINZADEH, N., SHOMALI, T., HOSSEINZADEH, S., et al., "Green synthesis of gold nanoparticles by using Ferula persica Willd . gum essential oil : production, characterization and in vitro anti-cancer effects" Pharmacy and Pharmacology, v. 72, n. 8, pp. 1-13, Apr. 2020.

[32] AZIZI, S., MOHAMAD, R., RAHIM, R.A., et al.“ZnO-Ag Core Shell Nanocomposite Formed by Green Method using Essential Oil of Wild Ginger and Their Bactericidal and Cytotoxic Effects" Applied Surface Science, v. 384, pp. 517-524, Oct. 2016.

[33] GA'AL, H., FOUAD, H., MAO, G. et al., "Larvicidal and pupicidal evaluation of silver nanoparticles synthesized using Aquilaria sinensis and Pogostemon cablin essential oils against dengue and zika viruses vector Aedes albopictus mosquito and its histopathological analysis" Artificial Cells, Nanomedicine, and Biotechnology, v. 64, n. 6, pp. 1171-1179, Sep. 2017.

[34] VILAS, V., PHILIP, D., MATHEW, J., "Essential oil mediated synthesis of silver nanocrystals for environmental, anti-microbial and antioxidant applications" Materials Science \& Engineering C, v. 61, pp. 429-436, Apr. 2016.

[35] SHENY, D.S., MATHEW, J., PHILIP, D. "Synthesis characterization and catalytic action of hexagonal gold nanoparticles using essential oils extracted from Anacardium occidentale" Spectrochimica Acta Part A: Molecular and Biomolecular Spectroscopy, v. 97, pp. 306-310, Nov. 2012.

[36] VILAS, V., PHILIP, D., MATHEW, J., "Biosynthesis of Au and Au/Ag alloy nanoparticles using Coleus aromaticus essential oil and evaluation of their catalytic, antibacterial and antiradical activities" Journal of Molecular Liquids, v. 221, pp. 179-189, Sep. 2016.

[37] MUNIYAPPAN, N., NAGARAJAN, N.S. “ Green synthesis of gold nanoparticles using Curcuma pseudomontana essential oil, its biological activity and cytotoxicity against human ductal breast carcinoma cells T47D" Journal of Environmental Chemical Engineering, v. 2, n. 4, pp. 2037-2044, Dec. 2014.

[38] SUTTHANONT, N., ATTRAPADUNG, S., NUCHPRAYOON, S., "Larvicidal Activity of Synthesized Silver Nanoparticles from Curcuma zedoaria Essential Oil against Culex quinquefasciatus" Insects, v. 10, n. 1, pp. 27, Jan, 2019.

[39] VILAS, V., PHILIP, D., MATHEW, J., "Catalytically and biologically active silver nanoparticles synthesized using essential oil” Spectrochimica acta part a: molecular and biomolecular spectroscopy, v. 
132, pp. 743-750, Nov. 2014.

[40] MANJU, S.,. MALAIKOZHUNDAN, B., VIJAYAKUMAR, S., et al.,, "Antibacterial , antibiofilm and cytotoxic effects of Nigella sativa essential oil coated gold nanoparticles" Microbial Pathogenesis, v. 91, pp. 129-135, Feb. 2016.

[41] VEISI, H., DADRES, N., MOHAMMADI, P., et al., "Green synthesis of silver nanoparticles based on oil-water interface method with essential oil of orange peel and its application as nanocatalyst for A 3 coupling" Materials Science \& Engineering C, v. 105, n. September 2018, p. 110031, Dec. 2019.

[42] ARASSU, R.R.T., NAMBIKKAIRAJ, B., RAMYA, D.R., "Green synthesis of silver nanoparticles and characterization using plant leaf essential oil rosemarinus officinalis and their antifungal activity against human pathogenic fungi” Journal of Scientific Research in Pharmacy, v. 7, n. 11, pp. 138-144, Nov. 2018.

[43] O. MACIEL, M.V., R. ALMEIDA, A. , MACHADO, M.H., et al., , "Syzygium aromaticum L . ( Clove ) Essential Oil as a Reducing Agent for the Green Synthesis of Silver Nanoparticles" Open Journal of Applied Sciences, pp. 45-54, Jan. 2019.

[44] PJ SARNOSKI, S.O., JOHNSON, J.V., REED, K.A., et al., "Separation and characterization of proanthocyanidins in Virginia peanut shells by LC - MS" Food Chemistry, vol. 131, pp. 927-939, Apr. 2012. [45] XY JIN, Z.C., LIU, Y., TAN, T., et al., "Cr (VI) removal from aqueous solutions via reduction and absorption by green synthesized iron nanoparticles" J. Clean. Prod, v. 176, pp. 929-936, Mar. 2018.

[46] AHMAD, A.M., WANI, T., MANZOOR, I.A., et al., "Biosynthesis structural characterization and antimicrobial activity of gold and silver" Colloids Surfaces B, v. 107, pp. 227-234, Jul. 2013.

[47] BISWAL A.K., AND MISRA, P.K., "Biosynthesis and characterization of silver nanoparticles for prospective application in food packaging and biomedical applications" Materials Chemistry and Physics, $\mathrm{p}$. 123014, Aug. 2020.

[48] KEAT, C. L., AZIZ, A., "Biosynthesis of nanoparticles and silver nanoparticles" Bioresources and Bioprocessing, v. 2, pp. 47-58, Dec. 2015.

[49] EL-SAYED, A.D. "Biosynthesis and comparative bactericidal activity of silver nanoparticles synthesized by Aspergillus flavus and Penicillium crustosum against the multidrugresistant bacteria" Journal of Microbiology and Biotechnology, PMID: 30086620, Jul. 2018.

[50] ABBASI, E., MILANI, M., AVAL, S.F. et al., "Silver nanoparticles: synthesis methods, bioapplications and properties" Critical Reviews in Microbiology, v. 42, pp. 173-180, Jun. 2016.

[51] EDISON T.J.I.., SETHURAMANM. G., "Instant green synthesis of silver nanoparticles using Terminalia chebula fruit extract and evaluation of their catalytic activity on reduction of methylene blue" Process Biochemistry, v. 47, pp. 1351-1327, Sep. 2012.

[52] ZUORRO, A., IANNONE, A., NATALI S., et al., "Green Synthesis of Silver Nanoparticles Using Bilberry and Red Currant Waste Extracts" Processes, v. 7, N. 4.,pp. 1-11, Apr. 2019.

[53] NISHANTHI, R., MALATHI, S., PAUL, S.J. et al., "Green synthesis and characterization of bioinspired silver, gold and platinum nanoparticles and evaluation of their synergistic antibacterial activity after combining with different classes of antibiotics" Materials Science \& Engineering C, v. 96, pp. 693-707, Mar. 2019.

[54] GUIMARÃES M.L., SILVA JR, F.A.G., COSTA, M.M., et al., "Green synthesis of silver nanoparticles using Ziziphus joazeiro leaf extract for production of antibacterial agents" Applied Nanoscience, v. 10, pp. 1073-1081, Apr. 2020.

[55] NAJAFPOOR, A, . NOROUZIAN-OSTAD, R., ALIDADI, H., et al., "Effect of magnetic nanoparticles and silver-loaded magnetic nanoparticles on advanced wastewater treatment and disinfection" Journal of Molecular Liquids, v. 303, p. 112640, Apr. 2020.

[56] DERTLI, E., MAYER, M.J., AND NARBAD, A., "Impact of the exopolysaccharide layer on biofilms , adhesion and resistance to stress in Lactobacillus johnsonii FI9785" BMC Microbiology, v. 15, n. 8, pp. 1-9, Feb. 2015 .

\section{ORCID}

Jarbas Freitas Amarante

Milena Lima Guimarães

Helinando Pequeno de Oliveira
https://orcid.org/0000-0003-0850-621X

https://orcid.org/0000-0002-0550-8730

https://orcid.org/0000-0002-7565-5576 